\title{
Immune Thrombocytopenia after Asymptomatic COVID-19 Infection: A Case Report
}

\author{
Umair Mubashir ${ }^{1}$, Behzad Amoozgar ${ }^{1}$, Shuvendu Sen ${ }^{1}$, Varun Kaushal ${ }^{1}$, and karim \\ Bayanzay $^{1}$
}

${ }^{1}$ Jersey Shore University Medical Center

June 4, 2020

\begin{abstract}
Here we discuss a case of a middle-aged COVID-19 positive (on serology) female who presented with no symptoms pertaining to COVID-19 but with ITP related manifestations. The patient did not respond to platelet transfusion, but consecutive immunoglobulin infusion and steroid therapy resulted in a complete resolution.
\end{abstract}

Immune Thrombocytopenia after Asymptomatic COVID-19 Infection: A Case Report

Umair Mubashir MD ${ }^{1}$, Behzad Amoozgar MD ${ }^{1,2}$, Varun Kaushal MD¹, Karim Bayanzay MD¹, and Shuvendu Sen MD, MS ${ }^{1}$

1- Jersey Shore University Medical Center, Perth Amboy Division

2- University of California, Berkeley, School of Public Health

Corresponding Author: Behzad Amoozgar MD; Email: behzad.amoozgar@berkeley.edu; Affiliation: Jersey Shore University Medical Center, Perth Amboy Division and University of California, Berkeley, School of Public Health; Mailing address: 530 New Brunswick Avenue Perth Amboy, New Jersey 08861

Keywords

Immune thrombocytopenia; Coronavirus disease 2019; COVID-19; Autoimmune

Key Clinical Message

Correlation between COVID-19 infection and ITP manifestation as a side effect.

\section{Introduction}

Comprehending the clinical presentation, adverse effects, and consequences of Coronavirus disease 2019 or COVID-19 are dynamically evolving. It is not uncommon for a viral infection to result in acquired thrombocytopenia by inducing autoantibodies production against platelet antigens which commonly manifests as secondary immune thrombocytopenia or ITP. ${ }^{1,2}$ However, its incidence, degree of severity, and resistance to treatment in the background of COVID-19 as the etiology are still unknown. On top of that, it is crucial to distinguish the ITP from other aetiologies of thrombocytopenia due to the differences in approach and management. Here we present a case of asymptomatic Covid-19 who presented only with symptomatic ITP.

\section{Case Presentation}

This is a case of a 58-year-old female with no significant past medical history who presented to emergency department with the chief complaint of bruising for the last 6 days. The patient had been in the postmenopausal state for the last 10 years. She was in her usual state of health 6 days before the current 
encounter when she noticed bruising in both arms. After self-administration of aspirin in hopes it would help with her symptoms, she developed bleeding from her gums and vaginal bleeding on the same day. She continued to have vaginal spotting and bleeding from her gums the following days. The patient denied any cough, chest pain, shortness of breath, joint pains, myalgias, recent travel, viral illness, or sick contact.

On the physical exam, the patient had ecchymosis in her bilateral upper extremities with the right arm worse than left. The areas of ecchymosis included $10 \mathrm{~cm}$ on the medial right arm and multiple small spots on the left arm. Moreover, petechia and bruises were appreciated on the patient's face and her inner buccal mucosa.

The patient's x-ray was normal. Initial labs showed platelet of less than $5^{*} 10^{3} / \mu \mathrm{L}$ (normal range: 150 -

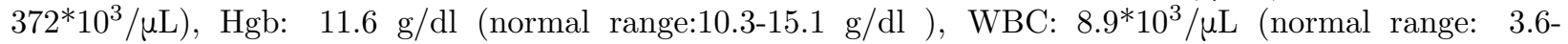
$\left.11^{*} 10^{3} / \mu \mathrm{L}\right)$, with a low absolute lymphocyte count $\left(1.310^{3} / \mu \mathrm{L}\right.$ and percentage of 14.3 ; normal range: 1.5-3.5* $10^{3} / \mu \mathrm{L}$ and 17-45\%), PTT: $23.6 \mathrm{sec}$ (normal range: $23-36 \mathrm{sec}$ ), INR: 0.98 (normal range: 0.9-1.1), and D-Dimer: $0.6 \mathrm{mg} / \mathrm{L}$ (normal range $<0.5 \mathrm{mg} / \mathrm{L}$ ). Routine chemistry was normal except for potassium of $3.4 \mathrm{mmol} / \mathrm{L}$ (normal $3.4-5.5 \mathrm{mmol} / \mathrm{L}$ ). Additionally, peripheral blood smear depicted neutrophilia and marked thrombocytopenia.

The hematologist was consulted and the related immunologic panels including platelet antibody, antinuclear antibodies (ANA), rheumatoid factor (RF), Lupus anticoagulant, antiphospholipid antibody (APA), serum protein electrophoresis (SPEP) were sent. In addition, due to the association of ITP with a viral infection, hepatitis B, C, HIV, and Covid-19 lab panels were ordered. Moreover, two units of platelets were transfused and solumedrol 1 gram/day was initiated, followed by $40 \mathrm{mg}$ every 8 hours.

The patient's platelet count did not improve $\left(6^{*} 10^{3} / \mu \mathrm{L}\right.$ after transfusion of two units PLT) and hence intravenous immunoglobulin or IVIG was started. Meanwhile, the ultrasonography of the spleen was reported normal. Patient platelets subsequently improved to $111^{*} 10^{3} / \mu \mathrm{L}$ (normal range: $150-372^{*} 10^{3} / \mu \mathrm{L}$ ) after a total of three units of platelet and two doses of IVIG administration. Later, HIV ab/ag, hepatitis panel, and antiphospholipid lab studies came back negative. However, platelet IgM antibody and Covid-19 RT PCR (nasopharyngeal sample) were positive.

The patient was discharged home with a taper prolonged dose of prednisone tablets (Deltasone $5 \mathrm{mg}$ tapered over 28 days) and did not report any new bleeding episodes or similar skin lesions during the telemedical follow-up visit.

\section{Discussion}

Mostly idiopathic, ITP is the result of platelet destruction by autoantibodies production, triggered by a non-apparent (primary ITP) or a secondary coexisting disorder (secondary ITP) $\cdot{ }^{1-4}$ Secondary acute ITP is mostly benign and can present with bleeding tendency, including purpura, and petechiae on skin and mucosal surfaces. Viral infections are among the major aetiologies of secondary acute ITP. ${ }^{5,6}$ In the background of viral infection, other contributory reductive mechanisms such as infection of megakaryocytes and decreases in thrombopoietin have previously been described in literature. ${ }^{7,8}$

Emerging of the novel coronavirus or COVID-19 has resulted in the occurrence of different types of inflammatory and auto immune disorders, either concomitantly or after the infection. ${ }^{9,10}$ The exact immunomodulatory mechanisms of Covid-19 infections which lead to platelet destruction it is currently under investigation. Recent studies postulated that COVID-19 directly infect bone marrow cell lines through CD13 receptors and induces apoptosis and growth inhibition in hematopoietic stem cells. Furthermore, COVID-19 may trigger cascades of immune responses that involve cytokines, $\mathrm{T}$ cells, and $\mathrm{B}$ cells that eventually result in autoantibody production against platelets and their progenitor cells. ${ }^{11,12}$

Recently an article by Zulfiqar et.al. reported ITP in an asymptomatic COVID-19 patient. ${ }^{13}$ Interestingly our case did not have any COVID-19 related signs or symptoms during the patient encounter and the viral infection was an incidental finding. Our patient responded well to the regular treatment including IVIG and steroids and reported complete resolution. 


\section{Conclusion}

Like other viral infections, there may a relation between contracting COVID-19 infection and ITP occurrence as an adverse immunologic consequence. The exact mechanism of ITP in the background of COVID-19 infections is still not well understood but it is highly possible that the overstimulation of T-cells and a consecutively high degree of cytokines production may trigger immunomodulatory pathways that result in platelets destruction. Further clinical studies are required to further investigate the causality of COVID-19 in ITP manifestation.

\section{Consent}

Written informed consent was obtained from the patient for publication purposes.

\section{Availability of data and material}

All the relevant data were included in the manuscript.

\section{Funding}

Authors acknowledge that they did not receive any funding for this study.

\section{Authors' contributions}

Behzad Amoozgar MD: data collection, preparation, and revision of the manuscript

Umair Mubashir MD: data collection, preparation, and revision of the manuscript

Varun Kaushal MD: revision of the manuscript

Karim Bayanzay MD: preparation and revision of the manuscript

Shuvendu Sen MD: preparation and revision of the manuscript

\section{Acknowledgments}

The authors declare that there is no conflict of interest regarding the publication of this paper. Authors confirms that no organization funded this study. Authors are the only people who have made substantive contributions to the study.

\section{References}

1- Cines, D. B., Bussel, J. B., Liebman, H. A., \& Luning Prak, E. T. (2009). The ITP syndrome: pathogenic and clinical diversity. Blood, The Journal of the American Society of Hematology, 113(26), 6511-6521.

2- Satoh, T., \& Kuwana, M. (2017). Differential Diagnosis: Secondary ITP. In Autoimmune Thrombocytopenia (pp. 97-105). Springer, Singapore.

3- LeVine, D. N., \& Brooks, M. B. (2019). Immune thrombocytopenia (ITP): Pathophysiology update and diagnostic dilemmas. Veterinary clinical pathology, 48, 17-28.

4- Despotovic, J. M., \& Bussel, J. B. (2019). Immune Thrombocytopenia (ITP). In Platelets (pp. 707-724). Academic Press.

5- Cines, D. B., \& Blanchette, V. S. (2002). Immune thrombocytopenic purpura. New England Journal of Medicine, 346(13), 995-1008.

6- Labarque, V., \& Van Geet, C. (2014). Clinical practice: immune thrombocytopenia in paediatrics. European journal of pediatrics, 173(2), 163-172.

7- Bordin, G., Ballare, M., Zigrossi, P., Bertoncelli, M. C., Paccagnino, L., Baroli, A., .. \& \& Inglese, E. (1995). A laboratory and thrombokinetic study of HCV-associated thrombocytopenia: a direct role of HCV in bone marrow exhaustion?. Clinical and experimental rheumatology, 13, S39-43. 
8- Louache, F., Bettaieb, A., Henri, A., Oksenhendler, E., Farcet, J. P., Bierling, P., .. \& \& Vainchenker, W. (1991). Infection of megakaryocytes by human immunodeficiency virus in seropositive patients with immune thrombocytopenic purpura.

9- Dogan, L., Kaya, D., Sarikaya, T., Zengin, R., Dincer, A., Akinci, I. O., \& Afsar, N. (2020). Plasmapheresis treatment in COVID-19-related autoimmune meningoencephalitis: Case series. Brain, Behavior, and Immunity.

10- FABRE, A., MORAND, A., \& URBINA, D. (2020). COVID-19 and Kawasaki Like Disease: The KnownKnown, the Unknown-Known and the Unknown-Unknown.

11- Xu, P., Zhou, Q., \& Xu, J. (2020). Mechanism of thrombocytopenia in COVID-19 patients. Annals of Hematology, 1-4.

12- Lippi, G., Plebani, M., \& Henry, B. M. (2020). Thrombocytopenia is associated with severe coronavirus disease 2019 (COVID-19) infections: A meta-analysis. Clinica Chimica Acta.

13- Zulfiqar, A. A., Lorenzo-Villalba, N., Hassler, P., \& Andrès, E. (2020). Immune thrombocytopenic purpura in a patient with COVID-19. New England Journal of Medicine, e43. 